\title{
Pharmacokinetic Behavior of Micafungin in Rats with Carbon Tetrachloride-Induced Acute Hepatic Failure
}

\author{
Hiroki Konishi, ${ }^{*, a}$ Masatomo Sudo, ${ }^{a, b}$ Masaki Sumi, ${ }^{a}$ Hiroaki MoriI,${ }^{a}$ Tokuzo MinouchI,${ }^{a}$ \\ Tachio Aimoto, ${ }^{b}$ and Akira YAMAJI ${ }^{a}$ \\ ${ }^{a}$ Department of Hospital Pharmacy, Shiga University of Medical Science; Seta, Otsu 520-2192, Japan: and ${ }^{b}$ Faculty of \\ Pharmaceutical Sciences, Setsunan University; 45-1 Nagaotoge-cho, Hirakata 573-0101, Japan. \\ Received August 30, 2004; accepted November 22, 2004
}

We examined the pharmacokinetic behavior of micafungin, a novel antifungal agent, in rats receiving carbon tetrachloride $\left(\mathrm{CCl}_{4}\right)$ at a single dose of $2.5 \mathrm{ml} / \mathrm{kg}$. There was no significant change in the total clearance $\left(C L_{\text {tot }}\right)$ in $\mathrm{CCl}_{4}$-treated rats, while the steady-state volume of distribution $\left(V d_{\mathrm{ss}}\right)$ was significantly increased by $\mathrm{CCl}_{4}$ treatment. Alteration in the serum unbound fraction of micafungin after $\mathrm{CCl}_{4}$ treatment was unlikely in light of the serum albumin, bilirubin, creatinine, and urea nitrogen. The increased $V d_{\text {ss }}$ was attributable to augmentation in the accessibility of micafungin to peripheral tissue without impairment of the intrinsic clearance, because slight enhancement of the tissue distribution of micafungin was confirmed following $\mathrm{CCl}_{4}$ treatment.

Key words micafungin pharmacokinetics; acute hepatic failure rat; carbon tetrachloride; tissue distribution; elimination clearance

Micafungin is a novel, semisynthetic antifungal lipopeptide with an echinocandin-like chemical structure. This agent exerts its fungicidal action by inhibiting biosynthesis of 1,3$\beta$-D-glucan, an essential component of the cell wall of most pathogenic fungi, leading to osmotic instability, and ultimately, cell rupture. ${ }^{1-3)}$ Micafungin demonstrates potent antifungal activities against Candida spp. and Aspergillus spp. in vitro, and its therapeutic efficacy has been confirmed in the treatment of infectious disease caused by these fungi species. ${ }^{4,5)}$ Because of its poor intestinal absorbability, micafungin is administered via an intravenous route in clinical practice. As urinary recovery of the unchanged form is less than $1 \%$ of the total dose, it is believed that micafungin is eliminated mainly by non-renal removal. ${ }^{6,7)}$ In view of the severity of disease conditions requiring antifungal therapy, micafungin is often administered to critically ill patients with hepatic damage. However, there have not been any reports regarding the impact of hepatic dysfunction on the disposition of micafungin, even though the total body clearance is probably dependent on the metabolic capacity of the liver.

In the present study, we examined the pharmacokinetic behavior of intravenously administered micafungin in acute hepatic failure in a rat model intoxicated with carbon tetrachloride $\left(\mathrm{CCl}_{4}\right)$.

\section{MATERIALS AND METHODS}

Reagents Standard solutions of micafungin and FR195743 (internal standard (IS) for HPLC) were generously donated by Fujisawa Pharmaceutical Co., Ltd. (Osaka, Japan). Sodium salt of micafungin for injection (Funguard $^{\mathrm{TM}}$, containing $50 \mathrm{mg}$ micafungin per a vial) and cyclosporine for injection (Sandimmun ${ }^{\mathrm{TM}}$, containing $250 \mathrm{mg}$ cyclosporine per $5 \mathrm{ml}$ ampoule) were purchased from Fujisawa Pharmaceutical Co., Ltd. and Novartis Pharma KK (Tokyo, Japan), respectively. $\mathrm{CCl}_{4}$ was obtained from Attest (Kyoto, Japan). All other chemicals and solvents were of the highest quality commercially available.

Experimental Animals and Treatment Male SpragueDawley rats were obtained from CLEA Japan, Inc (Tokyo,
Japan). The rats were acclimatized for at least $2 \mathrm{~d}$ before assignment to experimental groups at 5-6 weeks of age $(140-190 \mathrm{~g})$, and were housed in a clean room maintained at $23 \pm 2{ }^{\circ} \mathrm{C}$ with a relative humidity $55 \pm 10 \%$ and 12 -h light/dark cycle. They were allowed free access to regular animal diet and drinking water except when fasted for $24 \mathrm{~h}$ before administration of micafungin. Acute hepatic failure in rats was induced by intraperitoneal injection of $50 \% \mathrm{CCl}_{4}$ in olive oil $(5 \mathrm{ml} / \mathrm{kg}) 24 \mathrm{~h}$ before administration of micafungin. The rats used in this study were handled in accordance with the Guidelines for Animal Experimentation of Shiga University of Medical Science, and the experimental protocol was approved by the Animal Care and Use Committee of the Research Center for Animal Life Science of this institution.

Administration of Micafungin and Blood Collection Rats were anesthetized by ethyl ether, and a polyethylene cannula (outer diameter, $0.8 \mathrm{~mm}$; inner diameter, $0.5 \mathrm{~mm}$ ) was inserted into the left femoral artery for serial blood sampling. After arterial cannulation, the animals were kept in a Bollman-type restraining cage with free access to food and water. Micafungin solution $(6 \mathrm{mg} / \mathrm{ml})$ was prepared by dissolving Funguard ${ }^{\mathrm{TM}}$ with physiological saline, and was administered via the right saphenous vein at a dose of $6 \mathrm{mg} / \mathrm{kg}$. Blood samples were collected before micafungin administration, and $5 \mathrm{~min}, 10 \mathrm{~min}, 30 \mathrm{~min}$, and 1, 2, 4, 6, 8, 10, 20 and $24 \mathrm{~h}$ after administration. The plasma fractions were separated by centrifugation and stored at $-80{ }^{\circ} \mathrm{C}$ until assayed.

Administration of Cyclosporine and Blood Collection $0.1 \%$ cyclosporine solution prepared by diluting Sandimmun $^{\mathrm{TM}}$ with physiological saline was administered to rats via the saphenous vein at a dose of $1 \mathrm{mg} / \mathrm{kg}$. Blood samples were collected $15 \mathrm{~min}, 30 \mathrm{~min}$, and 1, 2, 4, 6 and $9 \mathrm{~h}$ after administration.

Determination of Tissue-to-Plasma Distribution of Micafungin Micafungin $(6 \mathrm{mg} / \mathrm{kg})$ was intravenously administered to $\mathrm{CCl}_{4}$-treated and control rats and then the animals were killed by severing a carotid artery under ether anesthesia $2 \mathrm{~h}$ after micafungin administration. After collecting blood samples, the liver, kidneys and lungs were quickly removed and weighed. These organs were subjected to freezing 
and thawing, and then homogenized with ice-cold physiological saline to prepare $10 \%$ tissue homogenates. Micafungin concentrations in plasma and tissues were measured as described below. The tissue-to-plasma distribution ratio of micafungin was calculated by dividing the respective tissue concentrations by the plasma concentration.

Measurement of Micafungin Concentration in Plasma and Tissue Homogenate Micafungin concentration in plasma and tissue homogenate was measured according to the fluorescence HPLC method of Yamato et al., ${ }^{8)}$ with some modifications. To $1-\mathrm{ml}$ plastic microcentrifuge tubes, $50 \mu \mathrm{l}$ of the plasma sample or tissue homogenate, $50 \mu 1$ of ethanol solution of FR195743 (IS) $(3 \mu \mathrm{g} / \mathrm{ml})$ and $50 \mu \mathrm{l}$ of acetonitrile were successively added. These solutions were mixed vigorously for about $10 \mathrm{~s}$ to remove plasma protein and tissue fragments, followed by centrifugation at $9000 \boldsymbol{g}$ for $5 \mathrm{~min}$. A $50-\mu 1$ aliquot of the supernatant was injected into the HPLC. The chromatographic equipment (Shimadzu Co., Ltd., Kyoto, Japan) consisted of a Model LC-10A liquid chromatograph, a Model SCL-10Avp system controller, and a Model RF-10A fluorometric detector. The analytes were separated on a Cosmosil $\mathrm{C}_{18}$ column $(250 \times 4.6 \mathrm{~mm}$, Attest, Kyoto $)$ using a mixture of $20 \mathrm{~mm}$ potassium dihydrogenphosphate : acetonitrile $(45: 55(\mathrm{v} / \mathrm{v}))$ as the mobile phase. The mobile phase was delivered at a flow rate of $1 \mathrm{ml} / \mathrm{min}$, and the column effluent was monitored at an excitation wavelength of $273 \mathrm{~nm}$ and an emission wavelength of $464 \mathrm{~nm}$. The retention times of micafungin and IS were $4.5 \mathrm{~min}$ and $6.4 \mathrm{~min}$, respectively, and micafungin was quantified using the peak area ratio compared with IS. The calibration curve for the quantification of plasma micafungin concentration was linear and passed through the origin with a correlation coefficient of 0.999 or better in a range from 0 to $25 \mu \mathrm{g} / \mathrm{ml}$. The coefficient of variation of repeated measurements using the micafungin-free pooled plasma spiked with known amounts of micafungin was less than 5\%, irrespective of the amount added.

Measurement of Cyclosporine Concentration in Whole Blood Blood cyclosporine concentration was determined by a fluorescence polarization immunoassay technique using the TDxFLx system according to the manufacturer's instructions (Abbott Laboratories, Tokyo, Japan).

Biochemical Assay The activities of alanine transaminase (ALT) and asparatate transaminase (AST) in plasma were measured by the pyruvate oxydase $/ N$-ethyl- $N$-(2-hydroxy-3-sulfopopyl)- $m$-toluidine/4-aminoantipyrine coupling method using a commercial reagent kit (KAINOS Laboratories, Inc., Tokyo, Japan). The plasma concentrations of albumin, bilirubin, creatinine, and urea nitrogen were measured by the bromocresol green method, the azobilirubin method, the creatinine amidohydrolase/sarcosine oxidase coupling method, and the urease/glutamate dehydrogenase coupling method, respectively, using a laboratory auto-analyzer.

Pharmacokinetic Analysis of Micafungin and $\mathbf{C y}$ closporine Standard pharmacokinetic parameters of micafungin were obtained by the model-independent moment method using a computer program, MOMENT(EXCEL). ${ }^{9)}$ The elimination rate constant at the terminal phase $(k e)$ was determined by linear regression of the log-linear portion of plots of blood concentration against time. The area under the plasma micafungin concentration versus time curve after administration $(A U C)$ was calculated by a linear trapezoidal ap- proximation from time zero to the last sampling point, with the addition of a correction term by extrapolation to infinity using the ratio of the last predicted concentration to the $k e$. The half-life $\left(t_{1 / 2}\right)$ was calculated using $\ln 2 / k e$. The total body clearance $\left(C L_{\text {tot }}\right)$ was determined by dividing the intravenous dose $(6 \mathrm{mg} / \mathrm{kg})$ by the $A U C$. The mean residence time $(M R T)$ was determined by dividing the area under the first moment curve by the $A U C$. The apparent volume of distribution at steady-state $\left(V d_{\mathrm{ss}}\right)$ was estimated by multiplying the $C L_{\text {tot }}$ and $M R T$.

Pharmacokinetic parameters for cyclosporine were obtained in a similar manner to the experiment with micafungin.

Statistical Analysis The results are expressed as the mean \pm S.D. Statistical comparison of means between the two groups was made using Student's $t$-test or Welch's $t$-test according to equality of variance of data distribution, and a $p$ value less than 0.05 was considered significant.

\section{RESULTS}

The biological characteristics of the control and $\mathrm{CCl}_{4-}$ treated rats are shown in Table 1 . In the $\mathrm{CCl}_{4}$-treated rats, the activities of ALT and AST in plasma were markedly elevated, and a slight elevation was also observed in the levels of creatinine, bilirubin and urea nitrogen. However, there were no significant differences in albumin level or body weight between the two rat groups.

Table 1. Biochemical Parameters of Control and $\mathrm{CCl}_{4}$-Treated Rats

\begin{tabular}{lcc}
\hline \multicolumn{1}{c}{ Parameters } & Control & $\mathrm{CCl}_{4}$-treated \\
\hline ALT $(\mathrm{KU})$ & $28.8 \pm 3.9$ & $165.7 \pm 68.8^{* *}$ \\
AST $(\mathrm{KU})$ & $51.7 \pm 7.3$ & $573.8 \pm 134.7^{* *}$ \\
Albumin $(\mathrm{g} / \mathrm{dl})$ & $2.7 \pm 0.2$ & $2.6 \pm 0.3$ \\
Creatinine $(\mathrm{mg} / \mathrm{dl})$ & $0.18 \pm 0.02$ & $0.26 \pm 0.02^{* *}$ \\
Urea nitrogen $(\mathrm{mg} / \mathrm{dl})$ & $14.4 \pm 1.7$ & $26.2 \pm 12.6$ \\
Bilirubin $(\mathrm{mg} / \mathrm{dl})$ & $0.1 \pm 0.0$ & $0.3 \pm 0.1^{*}$ \\
Body weight $(\mathrm{g})$ & $167.6 \pm 24.1$ & $167.2 \pm 24.5$ \\
\hline
\end{tabular}

Each value represents the mean \pm S.D. of 5 experiments. $* p<0.05, * * p<0.01$ compared with control.

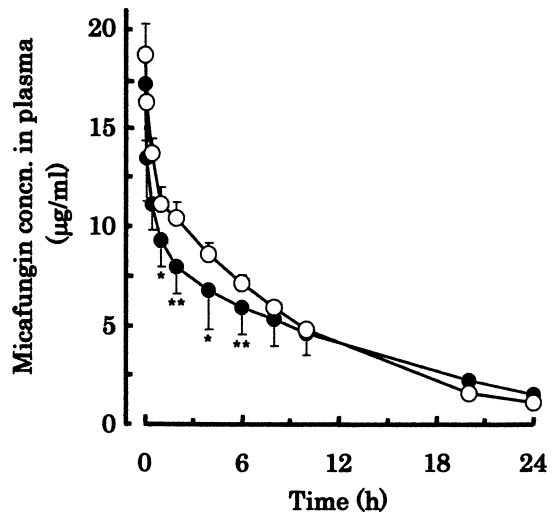

Fig. 1. Plasma Concentration-Time Courses of Micafungin after Intravenous Administration $(6 \mathrm{mg} / \mathrm{kg})$ to Control and $\mathrm{CCl}_{4}$-Treated Rats

Each point with a bar represents the mean \pm S.D. of 5 experiments. $* p<0.05$, $* * p<0.01$ compared with the corresponding value of control group. Symbols: $\bigcirc$, control rats; $\bullet, \mathrm{CCl}_{4}$-treated rats. 
Table 2. Pharmacokinetic Parameters of Micafungin of Control and $\mathrm{CCl}_{4}$ Treated Rats

\begin{tabular}{lcl}
\hline \multicolumn{1}{c}{ Parameters } & Control & $\mathrm{CCl}_{4}$-treated \\
\hline$k e(1 / \mathrm{h})$ & $0.105 \pm 0.011$ & $0.076 \pm 0.005^{* *}$ \\
$t_{1 / 2}(\mathrm{~h})$ & $6.60 \pm 0.69$ & $9.12 \pm 0.60^{* *}$ \\
$V d_{\mathrm{ss}}(1 / \mathrm{kg})$ & $0.418 \pm 0.031$ & $0.595 \pm 0.114^{*}$ \\
$C L_{\text {tot }}(1 / \mathrm{h} / \mathrm{kg})$ & $0.046 \pm 0.003$ & $0.047 \pm 0.009$ \\
$A U C(\mu \mathrm{g} \mathrm{h} / \mathrm{ml})$ & $131.3 \pm 8.1$ & $131.6 \pm 29.3$ \\
$M R T(\mathrm{~h})$ & $9.16 \pm 0.99$ & $12.67 \pm 1.13^{* *}$
\end{tabular}

Each value represents the mean \pm S.D. of 5 experiments. $* p<0.05, * * p<0.01 \mathrm{com}-$ pared with control.

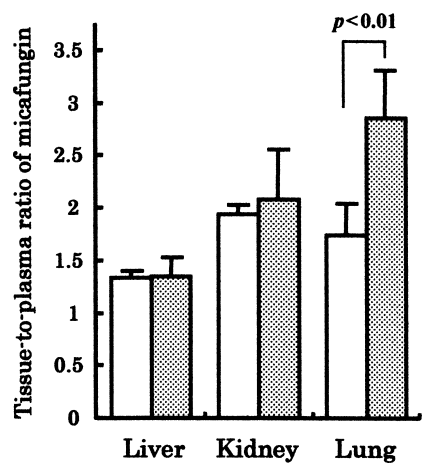

Fig. 2. Change in Tissue-to-Plasma Distribution Ratio of Micafungin after Treatment with $\mathrm{CCl}_{4}$

Liver, kidney and lung tissues were removed $2 \mathrm{~h}$ after i.v. bolus administration of micafungin $(6 \mathrm{mg} / \mathrm{kg})$ to control and $\mathrm{CCl}_{4}$-treated rats. Each column with a bar represents

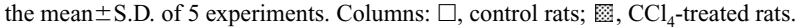

Figure 1 shows the plasma micafungin concentration-time curves in the control and $\mathrm{CCl}_{4}$-treated rats after intravenous administration of micafungin. In both groups, the plasma disappearance of micafungin after the end of the initial distribution phase followed an exponential decline. In the $\mathrm{CCl}_{4}$ treated rats, the plasma micafungin concentrations tended to fall in the early phase after administration and then approached the levels in the control rats during the late phase. The pharmacokinetic parameters in the two groups are shown in Table 2. In $\mathrm{CCl}_{4}$-treated rats, ke decreased by approximately $30 \%$ and $V d_{\text {ss }}$ and MRT increased by about $30 \%$. However, the $C L_{\text {tot }}$ remained unchanged.

In order to examine whether hepatic drug-metabolizing capacity was impaired by $\mathrm{CCl}_{4}$ treatment, we compared the pharmacokinetic parameters of intravenously administered cyclosporine in $\mathrm{CCl}_{4}$-treated rats $(n=4)$ with those in untreated rats $(n=4)$. The $C L_{\text {tot }}$ for cyclosporine in $\mathrm{CCl}_{4}$-treated rats was $37 \%$ lower than that in untreated rats $(p<0.05)$, but there was no significant difference in the $V d_{\text {ss }}$ between the two groups (data not shown).

Figure 2 shows the changes in the tissue distribution profile of micafungin after $\mathrm{CCl}_{4}$ treatment. In the $\mathrm{CCl}_{4}$-treated rats, a significant increase was observed in the lung-toplasma distribution ratio, although there were no significant changes in the ratios for the liver and kidney.

\section{DISCUSSION}

Acute exposure to $\mathrm{CCl}_{4}$ produces rapid cellular injury and centrilobular necrosis of the liver due to free radical-gener- ated lipid peroxidation of the hepatocyte membrane. Thus, $\mathrm{CCl}_{4}$-treated rats are widely used as common pathological models of acute hepatic failure in pharmacokinetic experiments. ${ }^{10-18)}$ Marked elevation in the activities of ALT and AST was confirmed in $\mathrm{CCl}_{4}$-treated rats, indicating the development of liver injury. $\mathrm{CCl}_{4}$ intoxication is involved in reducing hepatic blood flow as well as the activities of hepatic enzymes responsible for drug metabolism, ${ }^{14,19)}$ and it has been reported that the elimination clearance of many drugs that undergo hepatic removal is markedly reduced in $\mathrm{CCl}_{4^{-}}$ treated rats. ${ }^{10-14,17,18)}$

Micafungin is extensively biotransformed to a number of metabolites which are excreted in the urine and bile. ${ }^{6,7)}$ This property indicates that the disposition of micafungin is highly dependent upon a non-renal elimination process. In the present examination, it was shown that the $V d_{\mathrm{ss}}$ was significantly increased by acute hepatic failure, while the $C L_{\text {tot }}$ of micafungin was not affected. In general, this phenomenon is explained by an increase in the protein-unbound fraction of drug in serum, that is, the $C L_{\text {tot }}$ is assumed to be unchanged due to mutual cancellation of the reduced intrinsic metabolic clearance and the increased unbound drug concentration. Although the protein-unbound proportion of micafungin is presumed to be less than $0.3 \%,{ }^{20)}$ a validated method for quantitative measurement of the unbound micafungin concentration has not been established because of adsorption to experimental instruments and materials as well as limitations in the detection sensitivity. Instead, we examined the changeability of albumin and bilirubin as biological factors affecting proteinbinding capacity after $\mathrm{CCl}_{4}$ treatment. However, the degrees of their changes were minimal, and the accumulation of uremic toxins acting as protein-binding displacers was unlikely in light of only a slight elevation of creatinine and urea nitrogen. In addition, micafungin was reported to be resistant to the protein-binding displacement. ${ }^{20)}$ In $\mathrm{CCl}_{4}$-treated rats, the $C L_{\text {tot }}$ of cyclosporine was significantly reduced although the degrees of elevation of ALT and AST activities were not as prominent as expected. Significant reduction in the $C L_{\text {tot }}$ of cyclosporine is indicative of impairment of drug-metabolizing capacity in the liver, because this agent is eliminated exclusively by hepatic metabolism. ${ }^{21)}$ Judging from these findings, it appears appropriate to consider that, contrary to general belief, a significant prolongation in the half-life of micafungin is due more to an increase in the $V d_{\text {ss }}$ rather than to a decrease in intrinsic clearance. A likely explanation for this discrepancy is the involvement of multiple metabolic pathways independent of the decreased blood flow. Although many enzyme systems including sulfatase, catechol- $O$ methyltransferase and various CYP species have been clarified to contribute to micafungin biotransformation, ${ }^{6,7)}$ most metabolites and their metabolic routes remain unidentified. In this regard, the possibility of extrahepatic removal of micafungin cannot be ruled out, because this event is probably not directly associated with hepatic function.

It has been reported using rats that micafungin rapidly distributes into peripheral tissues such as liver, kidney and lung, and that micafungin in these tissues disappears in parallel with that in plasma. ${ }^{22}$ The increased $V d_{\mathrm{ss}}$ of micafungin in $\mathrm{CCl}_{4}$-treated rats is an interesting observation, since the $V d_{\text {ss }}$ of drugs is, in general, almost unaltered ${ }^{11,14,18)}$ or decreased $^{13)}$ probably due to impairment of blood supply to peripheral tis- 
sue. In this connection, we assumed that the augmented uptake of micafungin to tissues would lead to an increase in the $V d_{\mathrm{ss}}$ regardless of the change in unbound fraction. To test this hypothesis, we determined the tissue-to-plasma distribution ratio of micafungin $2 \mathrm{~h}$ after its administration, as it is expected that the distribution phase should already be completed at that time. There was no change in the amount distributed to the liver and kidney in the $\mathrm{CCl}_{4}$-treated rats, but the distribution ratio was markedly increased with respect to the lung tissue. This finding provides an explanation for the increased $V d_{\mathrm{ss}}$, although a reason for the differential degree of micafungin delivery among the organs is unknown. In a study using tumor-bearing rats, it was demonstrated that the tissue distribution profile of paclitaxel was markedly altered after $\mathrm{CCl}_{4}$ treatment, and that the lung-to-plasma distribution ratio of paclitaxel in $\mathrm{CCl}_{4}$-treated rats showed a propensity to become higher as compared with untreated rats. ${ }^{23)}$ These observations suggest that the apparent volume of distribution of drugs is likely to expand in some instances with $\mathrm{CCl}_{4}$-induced hepatic failure. It has been reported that exposure of rats to $\mathrm{CCl}_{4}$ causes oxidative damage to the lipids and proteins of lung as well as liver. ${ }^{24)}$ Therefore, the increased accessibility of micafungin to lung tissue may be attributed to pulmonary injury induced by the bioactivation of $\mathrm{CCl}_{4}$. Treatment with $\mathrm{CCl}_{4}$ should generate a systemic inflammatory response. In this regard, the increase in the $V d_{\mathrm{ss}}$ may also indicate the presence of a disease-dependent deep compartment and/or expansion of the extracellular space due to a rise in vascular permeability, in addition to enhancement of organ uptake.

In the present study using rats, it was demonstrated that the micafungin elimination clearance was not impaired by $\mathrm{CCl}_{4}$ treatment while the volume of distribution was significantly increased. Although the $A U C$ value of micafungin in $\mathrm{CCl}_{4}$-treated rats was similar to that in control rats, the degree of alteration in tissue micafungin concentration varied depending on the organ species. In view of a general need for pharmaceutical intervention against fungal infections in the critical care setting, we believe that it is also important to examine the pharmacokinetic behavior of micafungin under the experimental condition of more severe hepatic dysfunction.

Acknowledgements We would like to thank Dr. Kimie Imai and Mr. Naoto Inoue of the Faculty of Pharmaceutical Sciences, Setsunan University, for their technical support and helpful advice. We also thank Fujisawa Pharmaceutical Co., Ltd. for providing the standard solutions of micafungin and FR195743.

\section{REFERENCES}

1) Kurtz M. B., Heath I. B., Marrinan J., Dreikorn S., Onishi J., Douglas C., Antimicrob. Agents Chemother, 38, 1480-1489 (1994).

2) Tomishima M., Ohki H., Yamada A., Takasugi H., Maki K., Tawara S., Tanaka H., J. Antibiot., 52, $674-676$ (1999).

3) Hatano K., Morishita Y., Nakai T., Ikeda F., J. Antibiot., 55, 219-222 (2002).

4) Ikeda F., Folia Pharmacol. Jpn., 122, 339-344 (2003).

5) Jarvis B., Figgitt D. P., Scott L. J., Drugs, 64, 969-982 (2004).

6) Kaneko H., Yamato Y., Teramura Y., Fujiwara T., Suzuki A., Kawamura A., Terakawa M., Kagayama A., Jpn. J. Chemother., 50 (Suppl. 1), 88-93 (2002).

7) Yamato Y., Kaneko H., Yamasaki S., Fujiwara T., Katashima M., Kawamura A., Terakawa M., Kagayama A., Jpn. J. Chemother, 50 (Suppl. 1), 80-87 (2002).

8) Yamato Y., Kaneko H., Tanimoto K., Katashima M., Ishibashi K., Kawamura A., Terakawa M., Kagayama A., Jpn. J. Chemother, 50 (Suppl. 1), 68-73 (2002).

9) Tabata K., Yamaoka K., Kaibara A., Suzuki S., Terakawa M., Hata T., Xenobio. Metabol. Dispos., 14, 286-293 (1999).

10) Terblanche J., Hickman R., Dig. Dis. Sci., 36, 770_-774 (1991).

11) Shibata N., Shimakawa H., Minouchi T., Yamaji A., Biol. Pharm. Bull., 16, 1130-1135 (1993).

12) Fukuyama T., Yamaoka K., Tabata K., Nakagawa T., Biol. Pharm. Bull., 18, 251-255 (1995).

13) Ogiso T., Kitagawa T., Iwaki M., Tanino T., Biol. Pharm. Bull., 20, 405-410 (1997).

14) Sasa H., Hashimoto Y., Shimizu T., Inui K., Biol. Pharm. Bull., 21, 610-614 (1998).

15) Naora K., Ichikawa N., Hirano H., Iwamoto K., J. Pharm. Pharmacol., 51, 609-616 (1999).

16) Mukai T., Mera K., Nishida K., Nakashima M., Sasaki H., Sakaeda T., Nakamura J., Biol. Pharm. Bull., 25, $1494-1497$ (2002).

17) Taira Z., Yabe K., Hamaguchi Y., Hirayama K., Kishimoto M., Ishida S., Ueda Y., Food Chem. Toxicol., 42, 803-807 (2004).

18) Mukai T., Mera K., Nishida K., Nakashima M., Sasaki H., Nakamura J., Biol. Pharm. Bull., 27, 595-597 (2004).

19) Azri S., Mata H. P., Gandolfi A. J., Brendel K., Adv. Exp. Med. Biol., 283, 669-674 (1991).

20) Kaneko H., Yamato Y., Hashimoto T., Ishii I., Shiraga T., Kawamura A., Terakawa M., Kagayama A., Jpn. J. Chemother, 50 (Suppl. 1), 94-103 (2002).

21) Maurer G., Loosli H. R., Schreier E., Keller B., Drug Metab. Dispos., 12, 120-126 (1984).

22) Niwa T., Yokota Y., Tokunaga A., Yamato Y., Kagayama A., Fujiwara T., Hatakeyama J., Anezaki M., Ohtsuka Y., Takagi A., Biol. Pharm. Bull., 27, 1154-1156 (2004).

23) Fujita H., Okamoto M., Takao A., Mase H., Kojima H., Jpn. J. Cancer Chemother, 21, 659-664 (1994).

24) Abraham P., Wilfred G., Cathrine S. P., Clin. Chim. Acta, 289, 177 179 (1999). 